\title{
1 The gut parasite Nosema ceranae impairs 2 olfactory learning in bumblebees
}

3

4 Tamara Gómez-Moracho ${ }^{1, *, a}$, Tristan Durand ${ }^{1, *}$, Mathieu Lihoreau ${ }^{1}$

5

$6{ }^{1}$ Research Center on Animal Cognition (CRCA), Center for Integrative Biology (CBI);

7 CNRS, University Paul Sabatier, Toulouse, France

$8 *$ These authors contributed equally to this work

$9 \quad{ }^{a}$ Author for correspondence: Tamara Gómez-Moracho (tamara.gomez@univ-tlse3.fr)

11 Abstract

12 Pollinators are exposed to numerous parasites and pathogens when foraging on flowers. These 13 biological stressors may affect critical cognitive abilities required for foraging. Here, we 14 tested whether exposure to Nosema ceranae, one of the most widespread parasite of honey 15 bees also found in wild pollinators, impacts cognition in bumblebees. We investigated 16 different forms of olfactory learning and memory using conditioning of the proboscis 17 extension reflex. Seven days after feeding parasite spores, bumblebees showed lower 18 performance in absolute and differential learning, and reversal learning than controls. Long19 term memory was also slightly reduced. The consistent effect of $N$. ceranae exposure across 20 different types of olfactory learning indicates that its action was not specific to particular 21 brain areas or neural processes. We discuss the potential mechanisms by which $N$. ceranae 22 impairs bumblebee cognition and the broader consequences for populations of pollinators.

23 Keywords : Bumble bees ; Bombus terrestris; learning and memory ; PER 
25

26

27

\section{Introduction}

Pollinators, such as bees, rely on a rich cognitive repertoire to collect pollen and nectar on flowers. These include associative learning and memories of floral traits like odours, shapes, colours, and textures, to identify profitable resources (Giurfa, 2015; Menzel, 2012), and spatial cues to navigate (Collett et al., 2013). Any disruption of these cognitive abilities by environmental stressors can considerably reduce the foraging performances of bees, ultimately compromising brood development and survival (Klein et al., 2017).

In particular, foraging bees are exposed to a number of parasites that can affect their physiology and behaviour (Gómez-Moracho et al., 2017). The microsporidia Nosema ceranae is one of the most prevalent parasites of bees worldwide with a large range of hosts including honey bees (Higes et al., 2006), bumblebees (Plischuk et al., 2009), solitary bees (Ravoet et al., 2014), but also other flower visitors like wasps (Porrini et al., 2017). Insects get infected by ingesting parasite spores from contaminated water or pollen (Higes et al., 2008), or during physical contacts with contaminated individuals (Smith, 2012). The spores invade the gut epithelial cells of the bee where they develop (Holt et al., 2013). Nosema degenerates the gut epithelium of the host (Higes et al., 2007), alters its metabolism (Mayack and Naug, 2009) and disrupts the immune response (Antúnez et al., 2009). In honey bees, this causes a disease (nosemosis) believed to contribute to colony collapse disorder (Cox-Foster et al., 2007).

Infected honey bees also show impaired navigation (Wolf et al., 2014) and increased flight activity (Dussaubat et al., 2013) suggesting their cognitive abilities are affected. Few studies have explored this possibility and their results so far are mixed (Bell et al., 2020; Charbonneau et al., 2016; Gage et al., 2018; Piiroinen et al., 2016; Piiroinen and Goulson, 2016). Most of them were conducted on honey bees using Pavlovian olfactory conditionings of the proboscis extension reflex (PER) in which harnessed bees are trained to associate an odour, or a combination of odours, with a sucrose reward (Takeda, 1961). Honey bees showed reduced absolute learning (when an odour is paired to a reward) between days 7 and 23 after parasite exposure ((Bell et al., 2020; Gage et al., 2018; Piiroinen and Goulson, 2016); but see (Charbonneau et al., 2016)). Short-term memory was impaired after 7 and 15 days (Gage et al., 2018), but not after 9 (Piiroinen and Goulson, 2016) or 23 days (Bell et al., 2020). Long- 
55

56

57

58

59

60

61

62

63

64

65

66

67

68

69

70

71

72

73

74

75

76

77

78

79

80

81

82

83

term memory was affected after 7 days but not after 15 days (Gage et al., 2018). Only two studies explored these effects on bumblebees. One suggests a slight impairment of absolute learning (Piiroinen and Goulson, 2016), and both report an absence of effect on memory (Piiroinen et al., 2016, Piiroinen and Goulson 2016). Note however that in these two studies less than $3 \%$ of the bumblebees exposed to the parasite showed signs of infection after the behavioural tests (i.e. PCR positive).

Given the expanding geographical distribution of $N$. ceranae worldwide (Klee et al., 2007) and its prevalence in domesticated and wild bees (Plischuk et al., 2009; Porrini et al., 2017; Ravoet et al., 2014), clarifying its influence on host cognition has become an urgent matter. In particular, other critical forms of learning, such as the ability to associate one of two odours with a reward (differential learning) and reverse this association (reversal learning) have so far been unexplored. These types of learning are essential to discriminate flowers. They involve different brain centers and may thus be more or less impacted by the parasite if its effect is specific (Boitard et al., 2015; Giurfa and Sandoz, 2012).

Here we build on a recently established method yielding high the rates of experimental infection by N. ceranae (Gómez-Moracho et al., 2021) to study the impact of the parasite on different cognitive tasks in bumblebees. We used PER conditioning to compare the olfactory learning and memory performances of control bumblebees, bumblebees exposed to the parasite, and bumblebees contaminated by the parasites (PCR positive) at seven days post exposure.

\section{Material and methods}

\subsection{Bumblebees}

We used bumblebees (B. terrestris) from 14 commercial colonies acquired from Biobest (Westerlo, Belgium). Before the experiments, we verified the absence of N. ceranae (MartínHernández et al., 2007), and other common parasites (N. bombi (Klee et al., 2006); Crithidia bombi (Schmid-Hempel and Tognazzo, 2010)) in a PCR using 15 bumblebees from each colony. We maintained bumblebees in their original colonies with ad libitum access to the 
84

86

87

88

89

90

91

92

93

94

95

96

97

98

99

100

101

102

103

104

105

106

107

108

109

110

111

112

syrup provided by the manufacturer and germ-free pollen (honey bee collected pollen exposed to UV light for 12 hours), in a room at $25 \pm 1^{\circ} \mathrm{C}$ under a $12 \mathrm{~h}$ light: $12 \mathrm{~h}$ dark photocycle.

\subsection{N. ceranae spores}

We obtained fresh spores from naturally infected honey bee colonies (Apis mellifera) maintained at our experimental apiary (University Toulouse III, France). To prepare spore solutions, we dissected the gut of 15 honey bees and crushed them in $15 \mathrm{~mL}$ of distilled $\mathrm{H}_{2} \mathrm{O}$. We confirmed by PCR the presence of $N$. ceranae and the absence of $N$. apis (another common parasite of honey bees) in each homogenate (Martín-Hernández et al., 2007), and purified them following standard protocols (Fries et al., 2013). We centrifuged homogenates in aliquots of $1 \mathrm{~mL}$ at 5,000 rpm for 5 minutes and re-suspended the pellet in $500 \mu \mathrm{L}$ of $\mathrm{dH}_{2} \mathrm{O}$ by vortexing. This was repeated three times to obtain a spore solution of $85 \%$ purity (Fries et al., 2013). We counted $N$. ceranae spores in an improved Neubauer haemocytometer (Cantwell, 1970) in a light microscope (x400) and adjusted the spore inoculum to 15,000 spores $/ \mu \mathrm{L}$ in $20 \%(\mathrm{w} / \mathrm{w})$ of sucrose solution. Spore solutions were used within the same week they were purified.

\subsection{Parasite exposure and experimental conditions}

We exposed bumblebees to $N$. ceranae as described in Gómez-Moracho et al., (2021). Briefly, we confined individual bumblebees in a Petri dish during $5 \mathrm{~h}$ without food. We then exposed some bumblebees to a $20 \mu \mathrm{L}$ drop of inoculum containing 300,000 N. ceranae spores. Control bumblebees received $20 \mu \mathrm{L}$ of sucrose solution $(20 \% \mathrm{w} / \mathrm{w})$. We only used bumblebees that consumed the entire drop of sucrose within the next $2 \mathrm{~h}$. We then allocated bumblebees into microcolonies of 20-25 individuals, containing a gravity feeder with $\mathrm{ad}$ libitum access to food (Kraus et al., 2019). Since diet can affect host-parasites relationships (Frost et al., 2008) we provided bumblebees an artificial diet with a protein to carbohydrates ratio of 1:207 previously shown to elicit highest $N$. ceranae prevalence in bumblebees (Gómez-Moracho et al., 2021). The diet was made with a fixed total amount of nutrients of $170 \mathrm{~g} / \mathrm{L}$ (protein + carbohydrates) and $0.5 \%$ of vitamin mixture for insects (Sigma, Germany). 
113 Carbohydrates were supplied as sucrose (Euromedex, France). Proteins consisted in a mixture

114 of casein and whey (4:1) (Nutrimuscle, Belgium) (Gómez-Moracho et al., 2021). We kept

115 bumblebee microcolonies in a room at $25 \pm 1^{\circ} \mathrm{C}$ with a $12 \mathrm{~h}$ light: $12 \mathrm{~h}$ dark photoperiod until

116 the behavioural tests. Every day, we renewed the diet and removed dead bumblebees.

117

118

119

120

122

123

124

125

126

127

128

130

131

132

133

134

135

136

137

138

\subsection{Behavioural experiments}

We tested the cognitive performances of bumblebees using PER at day 7 after parasite exposure. The day before the behavioural tests we kept diet to low levels $(\sim 200$ $\mu \mathrm{L} /$ bumblebee) to keep bumblebees motivated for PER experiments. Three hours before the behavioural tests we collected bumblebees, chilled them in ice for $5 \mathrm{~min}$ and restrained them in a modified $2 \mathrm{~mL}$ Eppendorf tube (hereafter, capsule) that we cut in length to fit each bumblebee (adapted from Toda et al., (2009); Figure 1A). Bumblebees were tested in the horizontal position and could slightly move inside the tube. We found these conditions better suited to perform PER experiments with bumblebees than the classical vertical harnessing used for honey bees (Giurfa and Sandoz, 2012), in which bumblebees appeared paralysed (unpublished data). Once in the capsule, we kept the bumblebees in the dark, in an incubator at $28^{\circ} \mathrm{C}$, with no access to food. All bumblebees that finished the conditioning protocols were kept at $-20^{\circ} \mathrm{C}$ for later analyses of their infection status through PCR.

\subsection{Sucrose responsiveness}

We tested the sucrose responsiveness of bumblebees to control for potential influences of $N$. ceranae on their reward perception or feeding motivation. We presented seven sucrose solutions to each bumblebee, from concentrations of $0 \%$ (pure water) to $60 \%(\mathrm{w} / \mathrm{w})$, with increments of $10 \%$ (Graystock et al., 2013). For each concentration, we touched the antennae of the bumblebee with a toothpick soaked in the corresponding sucrose solution to elicit PER. We presented solutions in an increasing concentration gradient with an inter-trial interval of 5 minutes between concentrations. We scored a positive response if bumblebees extended their proboscis after a solution presentation. We discarded bumblebees responding to water (i.e. $0 \%$ sucrose solution) to avoid the effect of thirst on sucrose responsiveness (Baracchi et al., 
142 2018). The sum of the number of PER responses divided by the number of concentration

143 tested formed the individual gustatory score (Scheiner et al., 2013).

144

145

146

147

148

149

150

151

152

153

154

155

156

157

158

159

160

161

162

163

164

165

166

167

168

169

170

\subsection{Conditioning experiments}

All experiments shared the same general protocol (Figure S1). An encapsulated bumblebee (Figure 1A) was placed $1.5 \mathrm{~cm}$ ahead of an automated conditioning setup (described in (Aguiar et al., 2018)) delivering a continuous stream of odourless air at $1.2 \mathrm{ml} / \mathrm{s}$ to which specific odours were selectively added (Raiser et al., 2017). We used two odorants as conditioned stimulus (CS): nonanal and phenylacetaldehyde (Palottini et al., 2018), in a 1:100 dilution in mineral oil. Before conditioning, we tested the responsiveness of bees to sucrose by touching both antennae with a toothpick soaked in $50 \%(\mathrm{w} / \mathrm{w})$ sucrose solution without allowing them to lick. Bumblebees extending their proboscis were considered motivated and kept for the experiments. Conditioning trials (Figure S1c) consisted in 15 seconds of odourless airflow, followed by 6 seconds of CS, and 3 seconds of unconditioned stimulus (US) (i.e. 50\% sucrose solution applied with a toothpick on the bumblebee's antennae), with 2 seconds of overlap between CS+US, and 20 seconds of odourless airflow (Aguiar et al., 2018). The inter-trial interval was 10 minutes. An air extractor was placed behind the bumblebee to prevent odorant accumulation during CS delivery. Bumblebees extending their proboscis within 3 seconds of US presentation (i.e. $2 \mathrm{~s}$ CS+US and 1s US) were allowed to lick the toothpick soaked in sucrose $(50 \%, \mathrm{w} / \mathrm{w})$. In unrewarded trials (see reversal learning and memory tests) no US was applied. We scored a conditioned response if the bumblebee extended its proboscis to the odour delivery before sucrose presentation (Figure S1c). Bumblebees that responded to the odour in the first conditioning trial were discarded from the analyses. We used conditioned responses to calculate three individual scores for each bumblebee, describing its performance during conditioning (i.e. acquisition score), at the end of conditioning (i.e. learning score) or during memory retrieval (i.e. memory score) (Monchanin et al., 2020; see details below). Exposed and control bumblebees were always conditioned in parallel. 
172 We tested the effects of $N$. ceranae on the ability of bumblebees to associate an odour with a 173 reward. This form of learning only requires peripheral brain centers, i.e. the antennal lobes

174 (Giurfa and Sandoz, 2012). We trained bumblebees in a spaced 3-trial absolute conditioning 175 learning (Figure S1a) that was shown to generate robust long-term memory in bees (Menzel et 176 al., 2001). We used the same rewarded odour (CS+) during training of a given bumblebee, but 177 both nonanal and phenylacetaldehyde were used as a CS+ for different bumblebees. Only 178 bumblebees that responded to the US in the three trials (i.e. efficient bumblebees) were kept 179 for the analyses (Table S2). For each of these bees we calculated an acquisition score (sum of 180 PER responses (i.e. 0-2) divided by the number of trials (i.e. 3)), and a learning score (1 if 181 they responded to $\mathrm{CS}+$ in the last trial or 0 ).

182 We tested memory retention in efficient bumblebees that made at least one conditioned 183 response in either one of the last two trials. We performed tests either $1 \mathrm{~h}$ (i.e. short-term 184 memory; STM) or 24 h (i.e. long-term memory; LTM) after the last acquisition trial. For tests 185 performed after $24 \mathrm{~h}$, bumblebees were fed until satiation with $50 \%(\mathrm{w} / \mathrm{w})$ sucrose solution 186 right after conditioning. In bees, LTM is dependent on protein synthesis whereas STM is not 187 (Menzel, 2001). Studying these two types of memories was thus a mean to explore whether 188 exposure to N. ceranae interfered with protein synthesis. We presented bumblebees the two 189 odorants without any reward: the odour used as a CS+ to test for memory formation, and the 190 second odour as a novel odorant (NOd), to control for potential generalization (Villar et al., 191 2020). For example, when nonanal was used as CS+, phenylacetaldehyde was used as a NOd, 192 and vice versa. Memory formation was considered when bumblebees extended their proboscis 193 in response to the CS+ but not to the NOd, and thus had a memory score of 1 (0 otherwise). 194 Just after the memory test, we tested the motivation of bumblebees by touching their antennae 195 with a toothpick soaked with $50 \%(\mathrm{w} / \mathrm{w}$ ) sucrose solution. Bumblebees that did not respond to 196 the US were discarded for the analyses (i.e. 14.06\% in LTM; Table S2). 
199 We tested the effects of $N$. ceranae on the ability of bumblebees to learn to discriminate two

200 odours and reverse the task. This form of learning involves a first phase of differential

201 conditioning that requires the antennal lobes but is not dependant on central brain centers, i.e.

202 mushroom bodies, and a second phase of reversal learning that requires both the antennal

203 lobes and the mushroom bodies (Boitard et al., 2015). In the differential learning phase (phase

204 1), we trained bumblebees to discriminate between two odours. This phase consisted in 10-

205 trial blocks (Figure S1b), five with each odour that was either paired with the US (A+) or

206 unpaired (B-), presented in a pseudo-random order. The rewarded and unrewarded odours

207 were randomized on different training days. Bumblebees that did not respond to US in two or

208 more trials were discarded for the analyses and for reversal phase. In the reversal learning

209 phase (phase 2) we trained bumblebees to invert the first learnt contingency. The reversal

210 phase started $1 \mathrm{~h}$ after the end of the differential phase. Here we trained bumblebees in 12

211 trial-blocks, six with each odour presented in a pseudo-random order. The previously

212 rewarded odour was not associated with a reward anymore (A-) while the previously

213 unrewarded odour became rewarded $(\mathrm{B}+)$. To start with the same level of learning, we

214 analysed reversal learning in bumblebees that responded to A- either in the first and/or second

215 presentation of this odour during the reversal phase (Table S3). We analysed the performance

216 of bumblebees in each phase separately by attributing them an acquisition score (sum of all

217 trials where the bee responded to CS+ but not to CS-, divided by the number of trials) and a

218 learning score (1 if the bumblebee responded to CS+ but not to CS- in the last trial) for each 219 phase.

\subsection{Infection status}

222 We assessed the infection status of bumblebees that finished the tests in a PCR using the 223 primers 218MITOC (Martín-Hernández et al., 2007). For each bumblebee, the entire gut was 224 extracted, homogenised in sterile $\mathrm{dH} 2 \mathrm{O}$ and vortexed with $2 \mathrm{~mm}$ glass beads (Labbox 225 Labware, Spain). Genomic DNA was extracted using Proteinase K (20 mg/mL; Euromedex, 226 France) and $1 \mathrm{mM}$ of Tris-EDTA Buffer $(\mathrm{pH}=8)$. A sample with $N$. ceranae spores was

227 included in each round of extraction as positive control. PCRs were performed with the Taq 228 Polymerase Direct Loading Buffer (5 U/ $\mu \mathrm{L}$; MP Biomedicals, CA) following manufacturer's 
229

230

231

232

233

234

235

236

237

238

239

240

241

242

243

244

245

246

247

248

249

250

251

252

253

254

255

256

257

instructions. We used a final volume of $25 \mu \mathrm{L}$ with $0.4 \mu \mathrm{M}$ of each pair of primers (MartínHernández et al., 2007), $200 \mu \mathrm{M}$ of dNTPs (Jena Biosciences, Germany), $0.48 \mu \mathrm{g} / \mu \mathrm{L}$ of BSA (Sigma, Germany) and $2.5 \mu \mathrm{L}$ of DNA sample. PCR reactions were carried out in a S1000 Thermal Cycler (Biorad, CA). Thermal conditions were $94{ }^{\circ} \mathrm{C}$ for $2 \mathrm{~min}, 35$ cycles of $94{ }^{\circ} \mathrm{C}$ for $30 \mathrm{~s}, 61.8^{\circ} \mathrm{C}$ for $45 \mathrm{~s}$ and $72{ }^{\circ} \mathrm{C}$ for $2 \mathrm{~min}$, and a final step of $72{ }^{\circ} \mathrm{C}$ for $7 \mathrm{~min}$. The length of PCR products (i.e. $218 \mathrm{pb}$ ) was checked in a 1.2\% agarose gel electrophoresis stained with SYBR Safe DNA Stain (Edvotek, Washington DC). Positive and negative controls of PCR were run in parallel. Based on the PCR results we classified bumblebees in three different infection statuses, i.e. control, exposed negative or exposed positive. Bumblebees that were not exposed to the parasite but nevertheless showed a positive result in PCR were excluded from the analyses (i.e. 6.26\%; 23 out of 367 control bumblebees; Tables S1, S2 and S3).

\subsection{Statistical analyses}

All analyses were conducted in RStudio (version 1.4.1106). We evaluated the effect of parasite exposure and infection on gustatory, acquisition, learning and memory scores. The gustatory scores and the acquisition scores (i.e. absolute and reversal conditioning) were standardized and analysed in a Generalized Linear Mixed Model (GLMM) (package [glmmTMB]; (Brooks et al., 2017)). The response to the first and last sucrose concentrations, as well as the learning and memory scores were analysed in a binomial GLMM (package [lme4]; (Bates et al., 2015)). In all models, bee infection status was used as a fixed factor, while bee identity and odorant were used as random factors. We performed Tukey post-hoc pairwise comparisons (package [multcomp]; (Hothorn et al., 2008)) to assess the relationship between the three bee statuses. Raw data are available online (doi:10.5281/zenodo.4376362).

\section{Results}

\subsection{Parasite exposure did not influence sucrose responsiveness}

We tested responsiveness to different sucrose concentrations in 73 bumblebees (43 controls, 20 exposed negative, 10 exposed positive; Table S1). Overall, $71.23 \%$ of the bumblebees 
258 responded to sucrose (Figures $1 \mathrm{~B}$ and $\mathrm{C}$ ). This percentage was similar across the three 259 infection statuses $\left(\mathrm{X}^{2}=2.236, \mathrm{df}=2, \mathrm{p}=0.326\right)$. Bumblebees of the three infection statuses 260 responded similarly to the lowest sucrose concentration $\left(\right.$ Figure $1 \mathrm{~B} ; \mathrm{GLMM}, \mathrm{X}^{2}=0.920, \mathrm{df}=$ $2612, p=0.631$ ), reached similar gustatory scores (Figure $1 C$; GLMM, $X^{2}=1.686, d f=2, p=$ 262 0.430) and similar final level of response to the highest sucrose concentration (Figure 1B; 263 GLMM, $\left.\mathrm{X}^{2}=0.217, \mathrm{df}=2, \mathrm{p}=0.897\right)$. Therefore, exposure to $N$. ceranae affected neither the 264 sucrose sensitivity nor the feeding motivation of bumblebees.

A

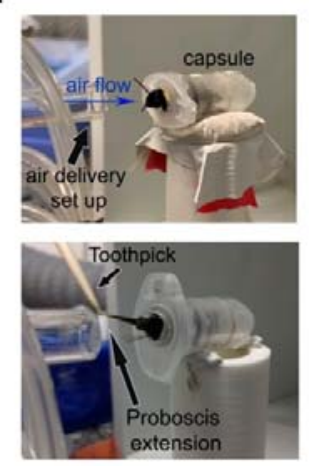

B

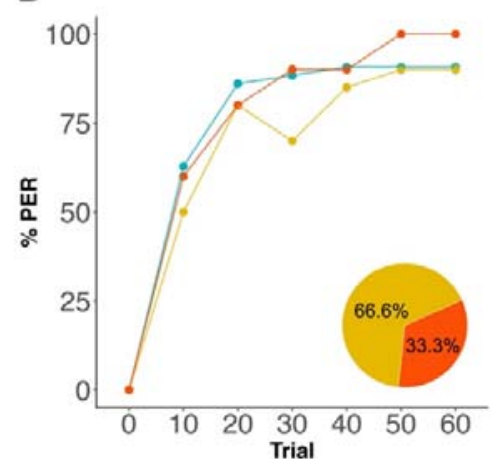

C

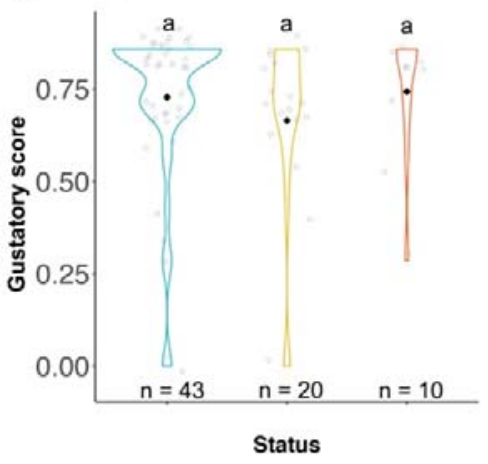

265

266

267

268

269

270

271

272

273

274

275

276

277

278

Figure 1. Odour delivery set up and sucrose responsiveness. A) Photo of a bumblebee during odour conditioning. The bumblebee is placed inside the capsule in front of the air delivery set up. After the odour is delivered, sucrose is presented with a toothpick to the bumblebees which extends its proboscis to drink the reward. B) Proportion of control (blue), exposed negative (yellow) and exposed positive (red) bumblebees responding to an increase gradient of sucrose concentrations. Pie chart represents the percentage of exposed negative and exposed positive bees ( $\mathrm{n}$ total=30). C) Violin plots show gustatory score of bumblebees as the sum of all responses for each bumblebee. Black diamonds represent the mean score for each infection status. White dots represent the score of each individual. $\mathrm{n}$ is the sample size. Letters above violin plots represent significant differences between status (GLMM; $p<0.05$ ). $\mathrm{n}$ is the sample size.

\subsection{Parasite exposure reduced absolute learning but not memory}


279 We analysed absolute conditioning in 420 bumblebees (141 controls, 228 exposed negative, 28051 exposed positive; Table S2). The proportion of bumblebees showing a conditioned 281 response increased over trials in the three infection statuses (Figure 2A). Exposed bumblebees 282 (either negative or positive) had significantly lower acquisition scores (Figure 2B; GLMM, 283 status: $\mathrm{X}^{2}=31.558, \mathrm{df}=2, \mathrm{p}<0.001$ ) and learning scores (Figure 2C; GLMM, status, $\mathrm{X}^{2}=$ 284 16.037, $\mathrm{df}=2, \mathrm{p}<0.001)$ than controls. Both acquisition and learning scores were similar for 285 negative and positive exposed bumblebees (Tukey $\mathrm{p}>0.05$; Table S4).
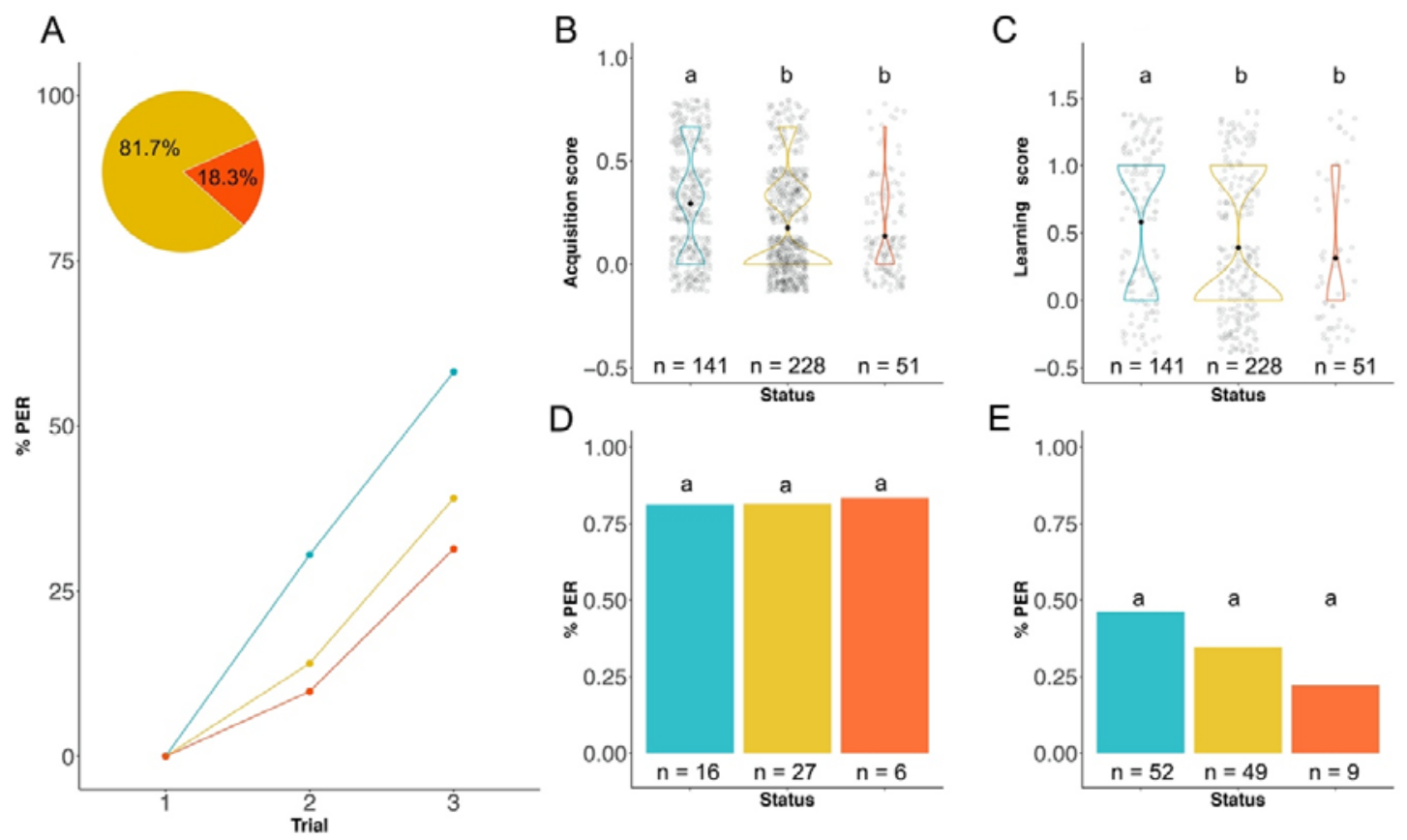

287 Figure 2. Absolute learning and memory. A) Learning curves show the proportion of control (blue), exposed negative (yellow) and exposed positive (red) bumblebees extending their proboscis to the odour during conditioning. Pie chart shows the percentage of negative and positive exposed bumblebees that finished the conditioning $(\mathrm{N}$ total $=279)$. B-C) Violin

291 plots for acquisition score (B; sum of correct responses divided by the number of trials for 292 each bumblebee) and learning score (C; correct response at the last trial). Black diamonds 293 represent the mean score for each infection status. White dots represent the score of each 294 individual. D) Short-term memory. Bar plots show the proportion of bumblebees responding 295 to odour $1 \mathrm{~h}$ after training. E) Long-term memory. Bar plots show the proportion of 
296 bumblebees responding to odour $24 \mathrm{~h}$ after training. Letters above violin plots and bar plots

297 represent significant differences between status (GLMM; $\mathrm{p}<0.05) . \mathrm{n}$ is the sample size.

299 We analysed short-term and long-term memory formation of efficient bumblebees that 300 responded to odour at least once in any of the last two last trials $(41.52 \%$ in short-term 301 memory and 50\% in long-term memory; Table S2). A high proportion of the bumblebees $302(82.02 \% \pm 0.011$, mean $\pm \mathrm{SE})$ remembered the rewarded odour $1 \mathrm{~h}$ after training. This proportion 303 was similar in the three infection statuses (GLMM, status: $X^{2}=0.013$, $\mathrm{df}=2, \mathrm{p}=0.993$ ), 304 indicating that $N$. ceranae did not affect short-term memory (Figure 2D). A much lower 305 proportion of the bumblebees $(34.35 \% \pm 0.06$, mean $\pm \mathrm{SE})$ remembered the rewarded odour 306 after $24 \mathrm{~h}$. Exposed bumblebees (either negative or positive) showed a lower proportion of 307 correct responses than controls, although this was not significantly different (Figure 2E; 308 GLMM, status: $\mathrm{X}^{2}=2.265$, df $=2, \mathrm{p}=0.322$ ), presumably due to the low amount of exposed 309 positive individuals (9 bumblebees).

\section{2. $\quad$ Parasite exposure reduced differential and reversal learning}

312 We analysed differential learning in 125 bumblebees (64 controls, 41 exposed negative, 20 313 exposed positive; Table S3). Bumblebees in the three infection statuses increased their 314 response to $\mathrm{A}+$ over trials, but not to $\mathrm{B}$ - (Figure 3A), indicating that they were equally able to 315 discriminate the odours. However, parasite exposure affected acquisition scores (GLMM, 316 status, $\mathrm{X}^{2}=22.039, \mathrm{df}=2, \mathrm{p}<0.001$; Figure 3B). Exposed bumblebees (positive or negative)

317 showed similar acquisition scores (Tukey test: $\mathrm{p}>0.05$; Table 1) and these scores were 318 significantly lower than the scores of controls (Tukey test: $p<0.05$; Table 1). Parasite 319 exposure also affected the final level of learning $\left(\mathrm{GLMM}\right.$, status, $\mathrm{X}^{2}=11.168, \mathrm{df}=2, \mathrm{p}=$ 320 0.003; Figure 2C). Exposed positive bumblebees had the lowest learning score. Their score 321 was significantly lower than that of controls (Tukey test: $\mathrm{p}=0.002$; Table 1) but similar to 322 that exposed negative bumblebees (Tukey test: $p=0.133$; Table 1). Thus overall, exposure to 323 N. ceranae reduced differential learning performances. 

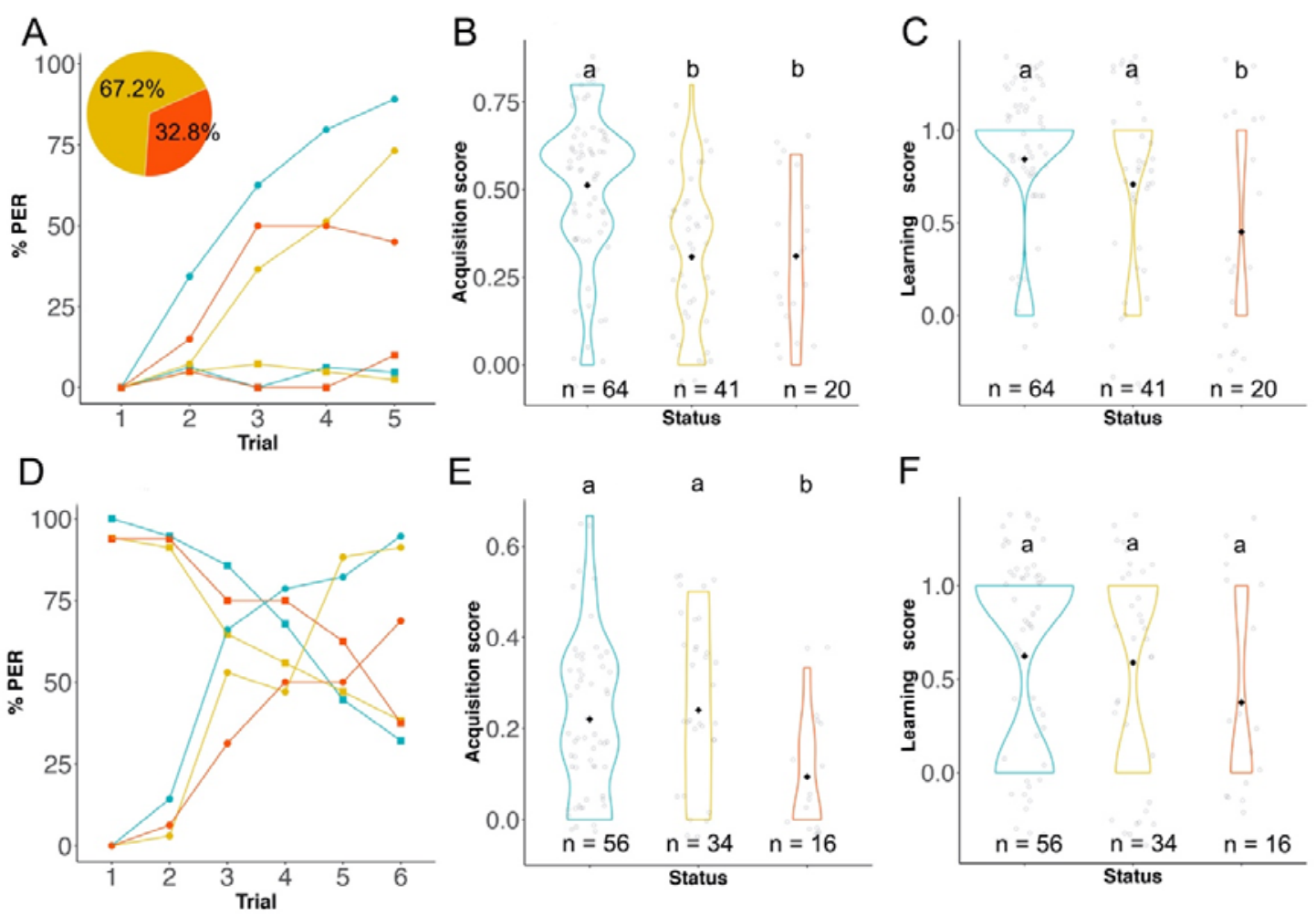

325 Figure 3. Reversal learning. A-C) Differential learning phase. A) Percentage of PER 326 responses to rewarded (A+, circle) and unrewarded (B-, square) odours by control (blue), exposed negative (yellow) and exposed positive (red) bumblebees. Pie chart shows the percentage of exposed negative and positive bumblebees that finished conditioning ( $\mathrm{n}$ total $=$ 67). Violin plots of B) acquisition scores (i.e. sum of the correct responses divided by the number of trials for each bee) and $\mathbf{C}$ ) learning scores (i.e. performance of bumblebees at last trial) of bumblebees with different infection statuses. Black diamonds represent the mean score of each status. White dots are the scores for each individual. D-F) Reversal learning phase. D) Curves show the increase in the percentage of PER response to B+over A- over trials. E) Acquisition score. F) learning scores. Letters above violin plots show significant differences between infection statuses in the acquisition and learning scores (GLMM, p < 0.05). $\mathrm{n}$ is the sample size. We analysed reversal learning in 108 bumblebees that finished the differential phase, as they responded to A- in any of the two first presentations of this odour during reversal phase (56 
340 controls, 36 exposed negative, 16 exposed positive; Table S3). All bumblebees reduced their

341 response to $\mathrm{A}$ - in favour of $\mathrm{B}+$ over trials (Figure 3D). However, bumblebees with different

342 infection statuses reached significantly different acquisition scores (Figure 3E; GLMM,

343 status, $\left.\mathrm{X}^{2}=6.042, \mathrm{df}=2, \mathrm{p}=0.048\right)$. Exposed positive bumblebees showed slightly lower

344 acquisition scores than controls (Tukey test: $\mathrm{p}=0.049$; Table 1) and exposed negative

345 bumblebees (Tukey test: $p=0.056$, Table 1), suggesting they had a lower ability to reverse

346 the task. By the end of training, bumblebees of all infection statuses reversed the task, as

347 shown by the lack of difference between their learning scores (Binomial GLMM, status: $\mathrm{X}^{2}=$

$3483.073, \mathrm{df}=2, \mathrm{p}=0.215)$. Thus overall, exposure to $N$. ceranae also impaired the reversal

349 phase of reversal learning. We found no effect of $N$. ceranae exposure on either phase of

350 reversal learning when bumblebees were tested at 2 days post exposure, suggesting that stress due to parasite exposure or parasite infection requires longer time to be established (Text S1).

352

353

354

355

356

357

358

359

360

361

362

363

364

365

366

367

368

369

\section{Discussion}

Bees are exposed to a number of parasites that can affect cognitive abilities supporting crucial behaviour (Koch et al., 2017; Schmid-Hempel, 2013). Previous studies exploring the effect of $N$. ceranae on absolute olfactory learning and memory in bees reported contrasting results, presumably because of differences in conditioning protocols and infection rates across studies (Bell et al., 2020; Charbonneau et al., 2016; Gage et al., 2018; Piiroinen et al., 2016; Piiroinen and Goulson, 2016). Here, we ran a suite of standard olfactory cognitive assays showing that feeding bumblebees spores of this parasite consistently impairs different types of olfactory learning but not memory seven days after exposure. This suggests that the influence of $N$. ceranae exposure on bumblebee cognition is not specific to a particular brain area.

Exposure to $N$. ceranae in food clearly impaired the ability of bumblebees to associate an odour with a reward (absolute learning), discriminate two odours (differential learning), and learn an opposite association (reversal learning). These are fundamental cognitive operations a bee must display to efficiently forage on flowers (Giurfa and Sandoz, 2012). This result agrees with a previous study reporting a reduced absolute learning in $N$. ceranae exposed bumblebees (Piiroinen and Goulson, 2016). Here we provide evidence that these reduced 
370 cognitive performances were not due to an effect of the parasite on odour perception or

371 motivation to feed, and give insight on where the parasite might act in the nervous system. In

372 bees, sucrose perception through the antennae, olfactory absolute learning, differential

373 learning and reversal learning all require processing of olfactory information through the

374 antennal lobes (Giurfa and Sandoz, 2012) while reversal learning also requires functional

375 mushroom bodies (Boitard et al., 2015). The fact that all types of learning were impaired and

376 that sucrose sensitivity was not, indicates that $N$. ceranae did not specifically target the

377 antennal lobes or the mushroom bodies. Rather, it likely impacted the learning processes in

378 general.

379 By contrast, we found no evidence that $N$. ceranae influenced memory. During training 380 animals learn and form short-term memories that are later consolidated and transformed into 381 stable long-term memories (Menzel and Muller, 1996) after protein synthesis (Menzel, 2001).

382 In our experiments, $N$. ceranae neither impaired short-term nor long-term memory. Nearly all 383 exposed bumblebees (either positive or negative in PCR) remembered the association of the 384 odour with the reward $1 \mathrm{~h}$ after training. However, they showed slightly lower performance 385 than controls at $24 \mathrm{~h}$. This tendency was not significant and may result from low sample size 386 as only $29.44 \%$ of the efficient exposed bumblebees were eligible to analyse their memory 387 (i.e. efficient bumblebees, with at least one conditioned response during learning, that 388 responded to sucrose after memory test).

389 It has recently been questioned whether bumblebees are natural hosts of $N$. ceranae based on 390 the lack of evidence of parasitic forms inside host cells (Gisder et al., 2020). Several studies 391 have nevertheless reported N. ceranae in wild bumblebees at low (e.g. 4.76\%; (Sinpoo, 392 2018)) and high prevalence (e.g. 72\%; (Arbulo et al., 2015)). Whether or not bumblebees are 393 suitable hosts for $N$. ceranae replication is confirmed in future studies, our results imply that 394 bumblebees are strongly impacted by an acute exposure to the parasite. Such exposure may be 395 extremely frequent in nature due to the high prevalence of $N$. ceranae in honey bees (Runckel 396 et al., 2011)) that contaminate flowers with spores through physical contact or in their faeces 397 (Graystock et al., 2015). Our protocol of parasite exposure significantly increased the 398 infection rate of bumblebees to $28 \%$ in comparison to previous studies (Piiroinen et al., 2016; 399 Piiroinen and Goulson, 2016), which allowed the evaluation of cognitive traits in bumblebees 
400 in the three infection statuses. Bumblebees that tested positive to $N$. ceranae showed a

401 tendency for lower cognitive performances than negative exposed bumblebees. They reached 402 the lowest learning during the absolute conditioning and barely discriminated odours, 403 suggesting that infection may interfere with some aspects of cognition, however further 404 experiments are needed to tackle this specific question.

405 Through which mechanism may the parasite impair learning? Ingestion of $N$. ceranae spores 406 exerts a stress that can reduce cognition. N. ceranae is known to alter the immune system of 407 bees, for example by modulating the expression of antimicrobial peptides (Antúnez et al., 408 2009; Botías et al., 2020; Sinpoo, 2018). Stimulation of the immune system with non409 pathogenic elicitors, as lipopolysaccharides (LPS), was shown to reduce learning abilities in 410 honey bees, that were less able to associate an odour with a reward (Mallon et al., 2003), and 411 bumblebees, that showed lower performances in odour (Mobley and Gegear, 2018) and colour 412 differential learning tasks (Mobley and Gegear, 2018). It is thus possible that the observed 413 effects of $N$. ceranae exposure on bumblebee cognition were caused by an activation of the 414 immune response. In honey bees, infection with $N$. ceranae was shown to downregulate the 415 expression of genes in the brain (Doublet et al., 2016), some of which are linked to olfaction 416 (Badaoui et al., 2017; Doublet et al., 2016), potentially leading to changes in behaviour and 417 cognition. Interestingly, none of these effects were observed at two days post exposure (Text $418 \mathrm{~S} 1)$. Whether $N$. ceranae triggers the immune response at this time is unknown. In honey 419 bees, the earliest effects described so far were observed after three days (Chaimanee et al., 420 2012).

421 Since olfactory learning is essential for foraging, this sublethal effect of $N$. ceranae exposure 422 on bumblebee cognition can compromise colony foraging and development. Commercial and 423 wild bumblebee colonies exhibit physiological and behavioural differences as a result of 424 different selective pressures (Velthuis and Doorn, 2006), and may, therefore, show different 425 susceptibility to parasites. Parasite loads in the field can range from a few to thousands spores 426 (Meana et al., 2010). Here we used a substantially higher spore loads of $N$. ceranae to infect 427 commercially reared bumblebees than the actual infection rates found in wild bumblebees 428 (e.g. 6800 spores per individual (Graystock et al., 2013)). Further studies are thus needed to 429 analyse the effects of different concentrations of $N$. ceranae spores, and their possible 
430 interactions with other stressors in the field in order to assess their real impact on wild 431 pollinators.

\section{Acknowledgements}

434

435 We thank Philipp Heeb, Cristian Pasquaretta and Maria Eugenia Villar for constructive 436 discussions, Charline Schartier for helping running some experiments, and Martin Giurfa for lending the automated PER conditioning setup.

438

\section{Funding}

440 TGM was funded by a postdoctoral grant of the Fyssen foundation. While writing, TGM, TD 441 and ML were funded by grants of the Agence Nationale de la Recherche (POLLINET ANR442 16-CE02-0002-01, 3DNaviBee ANR-19-CE37-0024, BEE-MOVE ANR-20-ERC8-0004-01), 443 the European Regional Development Fund (ECONECT), and the Agence de la Transition 444 Ecologique (LOTAPIS) to ML.

448 Aguiar, J.M.R.B.V., Roselino, A.C., Sazima, M., Giurfa, M., 2018. Can honey bees 449 discriminate between floral-fragrance isomers? J. Exp. Biol. 221, jeb180844. 450 https://doi.org/10.1242/jeb.180844

451 Antúnez, K., Martín-Hernández, R., Prieto, L., Meana, A., Zunino, P., Higes, M., 2009. 452 Immune suppression in the honey bee (Apis mellifera) following infection by Nosema 453 ceranae (Microsporidia). Environ. Microbiol. 11, 2284-2290. https://doi.org/10.1111/j.1462$454 \quad 2920.2009 .01953 . x$

455 Arbulo, N., Antúnez, K., Salvarrey, S., Santos, E., Branchiccela, B., Martín-Hernández, R., 456 Higes, M., Invernizzi, C., 2015. High prevalence and infection levels of Nosema ceranae in 457 bumblebees Bombus atratus and Bombus bellicosus from Uruguay. J. Invertebr. Pathol. 130, 
459 Badaoui, B., Fougeroux, A., Petit, F., Anselmo, A., Gorni, C., Cucurachi, M., Cersini, A., 460 Granato, A., Cardeti, G., Formato, G., Mutinelli, F., Giuffra, E., Williams, J.L., Botti, S., 461 2017. RNA-sequence analysis of gene expression from honeybees (Apis mellifera) infected 462 with Nosema ceranae. PLOS ONE $12, \quad$ e173438. 463 https://doi.org/10.1371/journal.pone.0173438

464 Baracchi, D., Rigosi, E., de Brito Sanchez, G., Giurfa, M., 2018. Lateralization of sucrose 465 responsiveness and non-associative learning in honeybees. Front. Psychol. 9, 425. 466 https://doi.org/10.3389/fpsyg.2018.00425

467 Bates, D., Mächler, M., Bolker, B., Walker, S., 2015. Fitting Linear Mixed-Effects models 468 using Ime4. J. Stat. Softw. 67. https://doi.org/10.18637/jss.v067.i01

469 Bell, H.C., Montgomery, C.N., Benavides, J.E., Nieh, J.C., 2020. Effects of Nosema ceranae 470 (Dissociodihaplophasida: Nosematidae) and flupyradifurone on olfactory learning in honey 471 bees, Apis mellifera (Hymenoptera: Apidae). J. Insect Sci. 20. 472 https://doi.org/10.1093/jisesa/ieaa130

473 Boitard, C., Devaud, J.-M., Isabel, G., Giurfa, M., 2015. GABAergic feedback signaling into 474 the calyces of the mushroom bodies enables olfactory reversal learning in honey bees. Front. 475 Behav. Neurosci. 9, 1-13. https://doi.org/10.3389/fnbeh.2015.00198

476 Botías, C., Jones, J.C., Pamminger, T., Bartomeus, I., Hughes, W.O.H., Goulson, D., 2020. 477 Multiple stressors interact to impair the performance of bumblebee (Bombus terrestris) 478 colonies. J. Anim. Ecol. 1365-2656.13375. https://doi.org/10.1111/1365-2656.13375

479 Brooks, M., E., Kristensen, K., Benthem, K., J. ,van, Magnusson, A., Berg, C., W., Nielsen, 480 A., Skaug, H., J., Mächler, M., Bolker, B., M., 2017. glmmtmb balances speed and flexibility 481 among packages for zero-inflated Generalized Linear Mixed Modeling. R J. 9, 378. 482 https://doi.org/10.32614/RJ-2017-066

483 Cantwell, G., 1970. Standard methods for counting Nosema spores. Am. Bee J. 110, 222-223. 
484 Chaimanee, V., Chantawannakul, P., Chen, Y., Evans, J.D., Pettis, J.S., 2012. Differential 485 expression of immune genes of adult honey bee (Apis mellifera) after inoculated by Nosema 486 ceranae. J. Insect Physiol. 58, 1090-1095. https://doi.org/10.1016/j.jinsphys.2012.04.016

487 Charbonneau, L.R., Hillier, N.K., Rogers, R.E.L., Williams, G.R., Shutler, D., 2016. Effects 488 of Nosema apis, N. ceranae, and coinfections on honey bee (Apis mellifera) learning and memory. Sci. Rep. 6, 22626. https://doi.org/10.1038/srep22626

490

Collett, M., Chittka, L., Collett, T.S., 2013. Spatial memory in insect navigation. Curr. Biol. 23, R789-R800. https://doi.org/10.1016/j.cub.2013.07.020

Cox-Foster, D.L., Conlan, S., Holmes, E.C., Palacios, G., Evans, J.D., Moran, N.A., Quan, P.493 L., Briese, T., Hornig, M., Geiser, D.M., Martinson, V., vanEngelsdorp, D., Kalkstein, A.L., 494 Drysdale, A., Hui, J., Zhai, J., Cui, L., Hutchison, S.K., Simons, J.F., Egholm, M., Pettis, J.S., 495 Lipkin, W.I., 2007. A metagenomic survey of microbes in honey bee Colony Collapse 496 Disorder. Science 318, 283-287. https://doi.org/10.1126/science.1146498

497 Doublet, V., Paxton, R.J., McDonnell, C.M., Dubois, E., Nidelet, S., Moritz, R.F.A., Alaux, 498 C., Le Conte, Y., 2016. Brain transcriptomes of honey bees (Apis mellifera) experimentally 499 infected by two pathogens: Black queen cell virus and Nosema ceranae. Genomics Data 10, 500 79-82. https://doi.org/10.1016/j.gdata.2016.09.010

501 Dussaubat, C., Maisonnasse, A., Crauser, D., Beslay, D., Costagliola, G., Soubeyrand, S., 502 Kretzchmar, A., Le Conte, Y., 2013. Flight behavior and pheromone changes associated to 503 Nosema ceranae infection of honey bee workers (Apis mellifera) in field conditions. J. 504 Invertebr. Pathol. 113, 42-51. https://doi.org/10.1016/j.jip.2013.01.002

505 Fries, I., Chauzat, M.-P., Chen, Y.-P., Doublet, V., Genersch, E., Gisder, S., Higes, M., 506 McMahon, D.P., Martín-Hernández, R., Natsopoulou, M., Paxton, R.J., Tanner, G., Webster, 507 T.C., Williams, G.R., 2013. Standard methods for Nosema research. J. Apic. Res. 52, 1-28. 508 https://doi.org/10.3896/IBRA.1.52.1.14

509 Frost, P.C., Ebert, D., Smith, V.H., 2008. Bacterial infection changes the elemental 510 composition of Daphnia magna. J. Anim. Ecol. 77, 1265-1272. 511 https://doi.org/10.1111/j.1365-2656.2008.01438.x 
512 Gage, S.L., Kramer, C., Calle, S., Carroll, M., Heien, M., DeGrandi-Hoffman, G., 2018.

513 Nosema ceranae parasitism impacts olfactory learning and memory and neurochemistry in

514 honey bees (Apis mellifera). J. Exp. Biol. 221, jeb161489. https://doi.org/10.1242/jeb.161489

515 Gisder, S., Horchler, L., Pieper, F., Schüler, V., Šima, P., Genersch, E., 2020. Rapid 516 gastrointestinal passage may protect Bombus terrestris from becoming a true host for Nosema 517 ceranae. Appl. Environ. Microbiol. 86. https://doi.org/10.1128/AEM.00629-20

518 Giurfa, M., 2015. Learning and cognition in insects: learning and insect cognition. Wiley 519 Interdiscip. Rev. Cogn. Sci. 6, 383-395. https://doi.org/10.1002/wcs.1348

520 Giurfa, M., Sandoz, J.-C., 2012. Invertebrate learning and memory: fifty years of olfactory 521 conditioning of the proboscis extension response in honeybees. Learn. Mem. 19, 54-66. 522 https://doi.org/10.1101/lm.024711.111

523 Gómez-Moracho, T., Durand, T., Pasquaretta, C., Heeb, P., Lihoreau, M., 2021. Artificial 524 diets modulate infection rates by Nosema ceranae in bumblebees. Microorganisms 9, 158. 525 https://doi.org/10.3390/microorganisms9010158

526 Gómez-Moracho, T., Heeb, P., Lihoreau, M., 2017. Effects of parasites and pathogens on bee 527 cognition: Bee parasites, pathogens and cognition. Ecol. Entomol. 42, 51-64. 528 https://doi.org/10.1111/een.12434

529 Graystock, P., Goulson, D., Hughes, W., 2015. Parasites in bloom: flowers aid dispersal and 530 transmission of pollinator parasites within and between bee species. Proc. R. Soc. B Biol. Sci. 531 282. https://doi.org/10.1098/rspb.2015.1371

532 Graystock, P., Yates, K., Darvill, B., Goulson, D., Hughes, W.O.H., 2013. Emerging dangers: 533 Deadly effects of an emergent parasite in a new pollinator host. J. Invertebr. Pathol. 114, 114534 119. https://doi.org/10.1016/j.jip.2013.06.005

535 Higes, M., García-Palencia, P., Martín-Hernández, R., Meana, A., 2007. Experimental 536 infection of Apis mellifera honeybees with Nosema ceranae (Microsporidia). J. Invertebr. 537 Pathol. 94, 211-217. https://doi.org/10.1016/j.jip.2006.11.001 
538 Higes, M., Martín, R., Meana, A., 2006. Nosema ceranae, a new microsporidian parasite in

539 honeybees in Europe. J. Invertebr. Pathol. 92, 93-95. https://doi.org/10.1016/j.jip.2006.02.005

540 Higes, M., Martín-Hernández, R., Botías, C., Bailón, E.G., González-Porto, A.V., Barrios, L., 541 del Nozal, M.J., Bernal, J.L., Jiménez, J.J., Palencia, P.G., Meana, A., 2008. How natural

542 infection by Nosema ceranae causes honeybee colony collapse. Environ. Microbiol. 10, 543 2659-2669. https://doi.org/10.1111/j.1462-2920.2008.01687.x

544 Holt, H.L., Aronstein, K.A., Grozinger, C.M., 2013. Chronic parasitization by Nosema 545 microsporidia causes global expression changes in core nutritional, metabolic and behavioral 546 pathways in honey bee workers (Apis mellifera). BMC Genomics 14, 799. 547 https://doi.org/10.1186/1471-2164-14-799

548 Hothorn, T., Bretz, F., Westfall, P., 2008. Simultaneous inference in general parametric 549 models. Biom. J. Biom. Z. 50, 346-363. https://doi.org/10.1002/bimj.200810425

550 Klee, J., Besana, A.M., Genersch, E., Gisder, S., Nanetti, A., Tam, D.Q., Chinh, T.X., Puerta, 551 F., Ruz, J.M., Kryger, P., Message, D., Hatjina, F., Korpela, S., Fries, I., Paxton, R.J., 2007.

552 Widespread dispersal of the microsporidian Nosema ceranae, an emergent pathogen of the 553 western honey bee, Apis mellifera. J. Invertebr. Pathol. 96, 1-10. 554 https://doi.org/10.1016/j.jip.2007.02.014

555 Klee, J., Tek Tay, W., Paxton, R.J., 2006. Specific and sensitive detection of Nosema bombi 556 (Microsporidia: Nosematidae) in bumble bees (Bombus spp.; Hymenoptera: Apidae) by PCR 557 of partial rRNA gene sequences. J. Invertebr. Pathol. 91, 98-104. 558 https://doi.org/10.1016/j.jip.2005.10.012

559 Klein, S., Cabirol, A., Devaud, J.-M., Barron, A.B., Lihoreau, M., 2017. Why bees are so 560 vulnerable to environmental stressors. Trends Ecol. Evol. 32, 268-278. 561 https://doi.org/10.1016/j.tree.2016.12.009

562 Koch, H., Brown, M.J., Stevenson, P.C., 2017. The role of disease in bee foraging ecology. 563 Curr. Opin. Insect Sci. 21, 60-67. https://doi.org/10.1016/j.cois.2017.05.008

564 Kraus, S., Gómez-Moracho, T., Pasquaretta, C., Latil, G., Dussutour, A., Lihoreau, M., 2019. 
565 Bumblebees adjust protein and lipid collection rules to the presence of brood. Curr. Zool. 65, 566 437-446. https://doi.org/10.1093/cz/zoz026

567 Mallon, E.B., Brockmann, A., Schmid-Hempel, P., 2003. Immune response inhibits 568 associative learning in insects. Proc. R. Soc. Lond. B Biol. Sci. 270, 2471-2473. 569 https://doi.org/10.1098/rspb.2003.2456

570 Martín-Hernández, R., Meana, A., Prieto, L., Salvador, A.M., Garrido-Bailon, E., Higes, M., 571 2007. Outcome of Colonization of Apis mellifera by Nosema ceranae. Appl. Environ. 572 Microbiol. 73, 6331-6338. https://doi.org/10.1128/AEM.00270-07

573 Mayack, C., Naug, D., 2009. Energetic stress in the honeybee Apis mellifera from Nosema 574 ceranae infection. J. Invertebr. Pathol. 185-188. 575 https://doi.org/10.1016/j.jip.2008.12.001

576 Meana, A., Martín-Hernández, R., Higes, M., 2010. The reliability of spore counts to 577 diagnose Nosema ceranae infections in honey bees. J. Apic. Res. 49, 212-214. 578 https://doi.org/10.3896/IBRA.1.49.2.12

579 Menzel, R., 2012. The honeybee as a model for understanding the basis of cognition. Nat. 580 Rev. Neurosci. 13, 758-768. https://doi.org/10.1038/nrn3357

581 Menzel, R., 2001. Searching for the memory trace in a mini-brain, the honeybee. Learn. Mem. 582 8, 53-62. https://doi.org/10.1101/lm.38801

583 Menzel, R., Muller, U., 1996. Learning and memory in honeybees: from behavior to neural 584 substrates. $\quad$ Annu. $\quad$ Rev. Neurosci. $\quad$ 19, 379-404. 585 https://doi.org/10.1146/annurev.ne.19.030196.002115

586 Menzel, Randolf, Manz, G., Menzel, Rebecca, Greggers, U., 2001. Massed and spaced 587 learning in honeybees: the role of cs, us, the intertrial interval, and the test interval. Learn. 588 Mem. 8, 198-208. https://doi.org/10.1101/lm.40001

589 Mobley, M.W., Gegear, R.J., 2018. Immune-cognitive system connectivity reduces 590 bumblebee foraging success in complex multisensory floral environments. Sci. Rep. 8, 5953. 
592 Monchanin, C., Drujont, E., Devaud, J.-M., Lihoreau, M., Barron, A.B., 2020. Heavy metal

593 pollutants have additive negative effects on honey bee cognition. bioRxiv 2020.12.11.421305.

594 https://doi.org/10.1101/2020.12.11.421305

595 Palottini, F., Estravis Barcala, M.C., Farina, W.M., 2018. Odor learning and its experience-

596 dependent modulation in the South American native bumblebee Bombus atratus

597 (hymenoptera: apidae). Front. Psychol. 9, 603. https://doi.org/10.3389/fpsyg.2018.00603

598 Piiroinen, S., Botías, C., Nicholls, E., Goulson, D., 2016. No effect of low-level chronic 599 neonicotinoid exposure on bumblebee learning and fecundity. PeerJ 4, e1808. 600 https://doi.org/10.7717/peerj.1808

601 Piiroinen, S., Goulson, D., 2016. Chronic neonicotinoid pesticide exposure and parasite stress 602 differentially affects learning in honeybees and bumblebees. Proc. R. Soc. B Biol. Sci. 283, 603 20160246. https://doi.org/10.1098/rspb.2016.0246

604 Plischuk, S., Martín-Hernández, R., Prieto, L., Lucía, M., Botías, C., Meana, A., 605 Abrahamovich, A.H., Lange, C., Higes, M., 2009. South American native bumblebees 606 (Hymenoptera: Apidae) infected by Nosema ceranae (Microsporidia), an emerging pathogen 607 of honeybees (Apis mellifera). Environ. Microbiol. Rep. 1, 131-135. 608 https://doi.org/10.1111/j.1758-2229.2009.00018.x

609 Porrini, M.P., Porrini, L.P., Garrido, P.M., de Melo e Silva Neto, C., Porrini, D.P., Muller, F., 610 Nuñez, L.A., Alvarez, L., Iriarte, P.F., Eguaras, M.J., 2017. Nosema ceranae in South 611 American native stingless bees and social wasp. Microb. Ecol. 74, 761-764. 612 https://doi.org/10.1007/s00248-017-0975-1

613 Raiser, G., Galizia, C.G., Szyszka, P., 2017. A high-bandwidth dual-channel olfactory 614 stimulator for studying temporal sensitivity of olfactory processing. Chem. Senses $42,141-$ 615 151. https://doi.org/10.1093/chemse/bjw114

616 Ravoet, J., De Smet, L., Meeus, I., Smagghe, G., Wenseleers, T., de Graaf, D.C., 2014. 617 Widespread occurrence of honey bee pathogens in solitary bees. J. Invertebr. Pathol. 122, 55- 
618 58. https://doi.org/10.1016/j.jip.2014.08.007

619 Runckel, C., Flenniken, M.L., Engel, J.C., Ruby, J.G., Ganem, D., Andino, R., DeRisi, J.L.,

620 2011. Temporal analysis of the honey bee microbiome reveals four novel viruses and seasonal

621 prevalence of known viruses, Nosema, and Crithidia. PLoS ONE 6, e20656.

622 https://doi.org/10.1371/journal.pone.0020656

623 Scheiner, R., Abramson, C.I., Brodschneider, R., Crailsheim, K., Farina, W.M., Fuchs, S.,

624 Grünewald, B., Hahshold, S., Karrer, M., Koeniger, G., Koeniger, N., Menzel, R., Mujagic,

625 S., Radspieler, G., Schmickl, T., Schneider, C., Siegel, A.J., Szopek, M., Thenius, R., 2013.

626 Standard methods for behavioural studies of Apis mellifera. J. Apic. Res. 52, 1-58.

627 https://doi.org/10.3896/IBRA.1.52.4.04

628 Schmid-Hempel, P., 2013. Evolutionary parasitology: the integrated study of infections, 629 immunology, ecology, and genetics. Oxford University Press.

630 Schmid-Hempel, R., Tognazzo, M., 2010. Molecular divergence defines two distinct lineages

631 of Crithidia bombi (Trypanosomatidae), parasites of bumblebees. J. Eukaryot. Microbiol. 57, 632 337-345. https://doi.org/10.1111/j.1550-7408.2010.00480.x

633 Sinpoo, C., 2018. Impact of Nosema ceranae and Nosema apis on individual worker bees of 634 the two host species (Apis cerana and Apis mellifera) and regulation of host immune 635 response. J. Insect Physiol. 8.

636 Smith, M.L., 2012. The honey bee parasite Nosema ceranae: transmissible via food 637 exchange? PLOS ONE 7, e43319. https://doi.org/10.1371/journal.pone.0043319

638 Takeda, K., 1961. Classical conditioned response in the honey bee. J. Insect Physiol. 6, 168639 179. https://doi.org/10.1016/0022-1910(61)90060-9

640 Toda, N.R.T., Song, J., Nieh, J.C., 2009. Bumblebees exhibit the memory spacing effect.

641 Naturwissenschaften 96, 1185-1191. https://doi.org/10.1007/s00114-009-0582-1

642 Velthuis, H.H.W., Doorn, A. van, 2006. A century of advances in bumblebee domestication 643 and the economic and environmental aspects of its commercialization for pollination. 
644 Apidologie 37, 421-451. https://doi.org/10.1051/apido:2006019

645 Villar, M.E., Marchal, P., Viola, H., Giurfa, M., 2020. Redefining single-trial memories in the

646 honeybee. Cell Rep. 30, 2603-2613.e3. https://doi.org/10.1016/j.celrep.2020.01.086

647 Wolf, S., McMahon, D.P., Lim, K.S., Pull, C.D., Clark, S.J., Paxton, R.J., Osborne, J.L.,

648 2014. So near and yet so far: harmonic radar reveals reduced homing ability of Nosema

649 infected honeybees. PLoS ONE 9, e103989. https://doi.org/10.1371/journal.pone.0103989

650 